\title{
Integrated aircraft and passenger recovery with cruise time controllability
}

\author{
Uğur Arıkan • Sinan Gürel • M. Selim Aktürk
}

Published online: 31 July 2013

(C) Springer Science+Business Media New York 2013

\begin{abstract}
Disruptions in airline operations can result in infeasibilities in aircraft and passenger schedules. Airlines typically recover aircraft schedules and disruptions in passenger itineraries sequentially. However, passengers are severely affected by disruptions and recovery decisions. In this paper, we present a mathematical formulation for the integrated aircraft and passenger recovery problem that considers aircraft and passenger related costs simultaneously. Using the superimposition of aircraft and passenger itinerary networks, passengers are explicitly modeled in order to use realistic passenger related costs. In addition to the common routing recovery actions, we integrate several passenger recovery actions and cruise speed control in our solution approach. Cruise speed control is a very beneficial action for mitigating delays. On the other hand, it adds complexity to the problem due to the nonlinearity in fuel cost function. The problem is formulated as a mixed integer nonlinear programming (MINLP) model. We show that the problem can be reformulated as conic quadratic mixed integer programming (CQMIP) problem which can be solved with commercial optimization software such as IBM ILOG CPLEX. Our computational experiments have shown that we could handle several simultaneous disruptions optimally on a four-hub network of a major U.S. airline within less than a minute on the average. We conclude that proposed approach is able to find optimal tradeoff between operating and passenger-related costs in real time.
\end{abstract}

Keywords Airline operations · Disruption management · Irregular operations · Passenger recovery $\cdot$ Cruise speed control $\cdot$ Conic quadratic mixed integer programming

U. Arıkan $(\varangle) \cdot$ S. Gürel

Department of Industrial Engineering, Middle East Technical University, Ankara, 06800, Turkey e-mail: arikanu@metu.edu.tr

S. Gürel

e-mail: gsinan@metu.edu.tr

M.S. Aktürk

Department of Industrial Engineering, Bilkent University, 06800 Bilkent, Ankara, Turkey

e-mail: akturk@bilkent.edu.tr 


\section{Introduction}

Air transportation has been an important industry for about one hundred years due to its economical and operational aspects. In this growing and highly competitive industry, airlines need to analyze a very complex and large-scale system in order to manage their resources efficiently while providing high-quality service to passengers. In the planning stage, the decisions of an airline are to select the flight legs to operate, determine the fleet type of each flight, assign aircraft and crew members to the flights. Gopalan and Talluri (1998) and Barnhart and Cohn (2004) describe optimization approaches for airline schedule planning in detail. However, schedules are rarely operated as planned. Original schedule often becomes infeasible due to disruptions such as bad weather conditions, unscheduled maintenance requirements and congestions at airports. When we analyze the data provided by Bureau of Transportation Statistics (BTS) (http://www.bts.gov), we observe that 12,899 of 46,571 flights (about $28 \%$ ) of a major U.S. airline experienced late arrivals in the first month of 2011. The average delay per flight was 33 minutes, which propagates and leads to infeasibilities in aircraft routings, crew schedules and passenger itineraries. Airline Operations Control Center (AOCC) is responsible for retrieving the initial schedule until the end of the day of operations. The options of AOCC controllers are to replan departure times of flights, cancel flights, swap aircraft, ferry aircraft and deadhead crew members, use reserve or standby crews, use standby aircraft and control the cruise speeds of aircraft within the limitations of aircraft capabilities. Disturbations in the original schedule and recovery actions result in huge costs; and hence, airlines require efficient and effective recovery methods.

Considering aircraft as the scarce resource, airlines typically create recovery plans for aircraft and passengers, sequentially. Most of the studies in the literature have focused on aircraft recovery; and integration with passenger recovery has not been explicitly considered in most of the recovery models. Jarrah et al. (1993) propose two minimum cost network flow models to recover from aircraft shortages. First model retimes departure times to minimize delay costs; and the second one determines cancelled flights in order to minimize cancellation costs. Thengvall et al. (2000) propose a flexible model allowing user preferences in the objective. The aim is to minimize total operating costs; however, passenger-related costs are not considered. Stojkovic et al. (2002) contribute by modeling and solving Day of Operations Scheduling problem optimally by modifications in arrival and departure times in real-time provided that the disruptions are minor. Rosenberger et al. (2003) present a model that reschedules flights and reroutes aircraft with the objective that minimizes rerouting and cancellation costs. The authors revise their model that attempts to maintain passenger connections by introducing a cost for disrupted itineraries.

Lan et al. (2006) propose two new approaches to minimize passenger disruptions and achieve robust airline schedule plans. The first approach involves aircraft routing and the second one involves retiming departure times. Aircraft routing problem is considered as a feasibility problem with the aim of achieving robustness with minimal cost implications. In the proposed robust aircraft maintenance routing (RAMR) model, the authors try to minimize the expected total propagated delay. In the second part of their study, the authors consider passengers who miss their flights due to insufficient connection time. Aim of this approach is to minimize the number of passenger misconnections by retiming the departure times of flights within a small time window. For retiming departure times, the authors propose the connection-based flight schedule retiming (CFSR) model. The objective of the model is to minimize the expected total number of disrupted passengers.

Bratu and Barnhart (2006) aim to find the optimal trade-off between airline operating costs and passenger delay costs, and propose two optimization models. The first one is 
named as Disrupted Passenger Metric (DPM) and aims to minimize passenger disruption costs without increasing operating costs. The second model is called Passenger Delay Metric (PDM) which minimizes passenger delay costs without increasing operating costs. Their experiments have shown that DPM is fast enough for making quick decisions, while PDM cannot be solved in real time.

Kohl et al. (2007) present a detailed overview of airline disruption management processes in practice. The authors also discuss the opportunity of avoiding high passenger delay costs by increasing cruise speeds of flights. In our study, we integrate cruise speed control in our alternative courses of actions and the results of our experimentation are in line with the authors' suggestions.

AhmadBeygi et al. (2008) analyze the potential for delays to propagate. In order to understand the relationship between aircraft schedules and delay propagation, authors investigate the flight data of two major U.S. airlines. They show that propagation due to a delayed flight has greater impact than the delay itself. It is observed that propagated delay more than doubles the initial root delay for flights that propagate delays even without considering connecting passengers. Jafari and Zegordi (2010) present an assignment model for recovering both aircraft and passengers simultaneously with the objective of minimizing the sum of aircraft assignment cost, total delay cost, cancellation cost and disrupted passenger cost. Proposed approach utilizes a wide range of recovery actions except for cruise speed control.

Similar to Bratu and Barnhart (2006), Marla et al. (2011) propose a formulation that utilizes time-space networks to solve integrated recovery problem. The authors contribute by incorporating cruise speed control, or flight planning, with time-space network successfully. Using flight planning together with other available recovery actions provides great reductions in passenger disruptions and overall disruption costs. However, integrating flight planning with time-space network representation requires a second dimension of discretization (flight options are discretized with respect to different departure times and different cruise speeds). Considering the size of airline networks, this representation allows several cruise speed options for each flight copy to be evaluated in the model. In our study, we aim to contribute by expressing cruise speed as a continuous variable and try to find the optimal tradeoff between increased fuel cost and other disruption costs by solving the resulting the nonlinear mixed integer formulation using an exact approach.

Petersen et al. (2012) study an integrated airline recovery problem using a single-day horizon, and propose a separate mixed-integer mathematical model for the schedule, aircraft, crew and passenger recovery problems. They utilize a Benders decomposition scheme together with the column generation approach to achieve the coordination among these four mathematical models. Since they place a 30-minute threshold of computation time for the overall problem, they also propose a sequential recovery algorithm to handle larger problems. For a recent review on airline disruption management, we refer to Clausen et al. (2010).

In disruption management literature, there is an increasing number of studies that tries to integrate passenger recovery with disruption management. On the other hand, there is also an increasing interest on modeling the passenger behavior. Recent studies try to integrate discrete choice models of passenger behavior with traditional revenue management, scheduling and other applications in aviation industry such as Garrow et al. (2010), Graham et al. (2010), and Garrow (2010).

In practice, recovery decisions are generally made considering the infeasibilities in aircraft routings and crew schedules; however, passengers are also severely affected. In resulting recovery plans, arrivals of passengers may be delayed or passenger itineraries may 
be disrupted; and hence, delay and spill costs are incurred. Delay costs are typically measured in dollars per minute delay of aircraft and per minute lost by each passenger. Barnhart et al. (2012) reported that in 2007, economic costs of time that passengers lost due to delays are estimated to be $\$ 12$ billion according to Joint Economic Committee report. Therefore, considering the disruptions in passenger itineraries in recovery process is crucial to reduce operating and inconvenience costs. In this paper, we propose a mathematical model for aircraft-passenger recovery problem which handles departure and arrival delays of any severity. Superimposition of aircraft and passenger networks is considered to create an integrated recovery plan. The objective is to minimize the total recovery cost which includes routing-related costs together with passenger-related costs. Passengers are discretized both by their itineraries and classes. We aim to contribute to the literature by proposing an integrated solution approach that reschedules departure and arrival times, swaps aircraft, determines passenger itineraries that will be disrupted and finds optimal cruise speeds of flights.

In addition to more commonly used recovery actions, we aim to contribute by including cruise speed control in the alternative courses of recovery actions. Aircraft can speed up to some extent and reduce cruise times as discussed in Cook et al. (2009). Our experiments have shown that cruise time reduction provides a great opportunity to mitigate delays. On the other hand, fuel consumption increases with cruise speed. In order to find the optimal tradeoff between fuel consumption and delay propagation, fuel cost is expressed as a nonlinear function of cruise time and included in the objective function. The problem is formulated as a mixed integer nonlinear programming (MINLP) model. Afterwards we show that the model can be represented as a conic quadratic mixed integer programming (CQMIP) problem. Finally, we generate conic quadratic constraints to solve the problem with commercial CQMIP solvers, such as CPLEX. We test our approach using publicly available schedules of a major U.S. airline. During our analysis of the executed airline schedules provided by BTS, we have found out that usually one or two disruptions are observed at a given time frame. Our computational experiments have shown that we could solve the integrated aircraft and passenger recovery problem to optimality on a four-hub network of a major U.S. airline in less than one minute on the average.

This paper is organized as follows. In Sect. 2, a numerical example is given for illustration. Details of the mathematical formulation are presented in Sect. 3. In Sect. 4, conic quadratic reformulation of MINLP model is discussed. Experimental setup, computational results and analysis of factors are presented in Sect. 5, and we conclude in Sect. 6.

\section{Numerical example}

Before presenting the mathematical formulation, we illustrate the main idea on a smallsized numerical example. The input of aircraft and passenger recovery problem consists of the flight schedule, characteristics of the assigned aircraft (such as seat capacities and fuel efficiencies), planned passenger itineraries, numbers of passengers of each class assigned to these itineraries, and lastly a set of disrupted flights with the severities of these disruptions. Flight schedule of the example is illustrated on a time-space network in Fig. 1. Vertical axis corresponds to space (airports) while the horizontal axis is the time line. Each line style (color) represents a different aircraft routing. The horizontal line segments of a routing represents the time that the aircraft spends at the airport. Length of these line segments need to be at least as long as the turn time of the aircraft. Turn time is the minimum time required after the arrival of an aircraft to be ready for its next departure. In the example problem, 


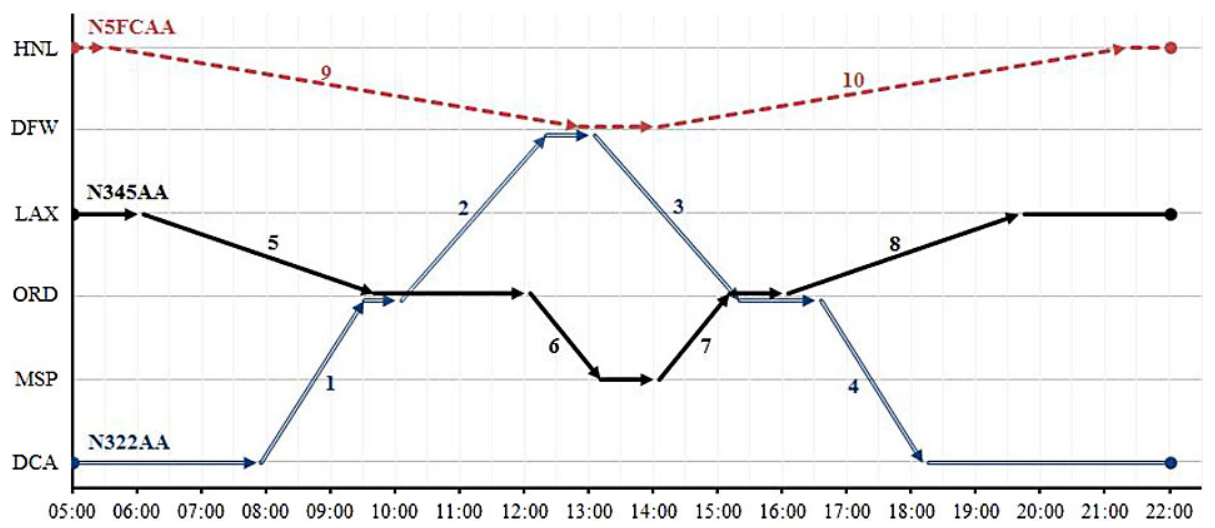

Fig. 1 Time-space network displaying the flight schedule of three aircraft routings

Table 1 Planned flight schedule

\begin{tabular}{lcllcrrrrr}
\hline Tail \# & Flight \# & From & To & $\begin{array}{l}\text { Departure } \\
\text { time }\end{array}$ & $\begin{array}{l}\text { Arrival } \\
\text { time }\end{array}$ & $\begin{array}{l}\text { Cruise } \\
\text { time } \\
\text { (min.) }\end{array}$ & $\begin{array}{l}\text { Time to } \\
\text { next } \\
\text { flight } \\
\text { (min.) }\end{array}$ & $\begin{array}{l}\text { Dist. } \\
\text { (miles) }\end{array}$ & $\begin{array}{l}\text { Allowable } \\
\text { compr. }\end{array}$ \\
\hline N322AA & 1 & DCA & ORD & $7: 50$ & $9: 30$ & 80 & 30 & 610 & $6 \%$ \\
& 2 & ORD & DFW & $10: 00$ & $12: 20$ & 120 & 40 & 800 & $6 \%$ \\
& 3 & DFW & ORD & $13: 00$ & $15: 20$ & 120 & 70 & 800 & $6 \%$ \\
& 4 & ORD & DCA & $16: 30$ & $18: 10$ & 80 & - & 610 & $6 \%$ \\
N345AA & 5 & LAX & ORD & $6: 00$ & $9: 40$ & 200 & 140 & 1745 & $10 \%$ \\
& 6 & ORD & MSP & $12: 00$ & $13: 10$ & 50 & 50 & 335 & $10 \%$ \\
& 7 & MSP & ORD & $14: 00$ & $15: 10$ & 50 & 50 & 335 & $10 \%$ \\
& 8 & ORD & LAX & $16: 00$ & $19: 40$ & 200 & - & 1745 & $10 \%$ \\
N5FCAA & 9 & HNL & DFW & $5: 30$ & $12: 50$ & 420 & 70 & 3780 & $9 \%$ \\
& 10 & DFW & HNL & $14: 00$ & $21: 20$ & 420 & - & 3780 & $9 \%$ \\
\hline
\end{tabular}

turn times for all flights are set to 30 minutes. Inclined line segments, on the other hand, correspond to planned flights. For instance, N5FCAA performs two flights: a 440-minute flight from HNL to DFW, and a 440-minute flight from DFW back to HNL. The aircraft spends 70 minutes at DFW between these flights.

Flight schedule is tabulated in Table 1. The rightmost column corresponds to the allowable compression percentage which is the aircraft-and-flight-dependent parameter indicating the allowable compression of flight time expressed in terms of the percentage of cruise time. For instance, cruise time, and hence the flight time, of flight 1 can be decreased by 4.8 minutes at most, if the aircraft of the flight is not swapped.

Passenger itineraries expressed in terms of sequential flight numbers are listed in Table 2 (the second and third columns represent the numbers of passengers in economy and business classes, respectively); and space network of flights and passenger itineraries are represented in Fig. 2. It is important to note that we deal with the integrated network, which is the superimposition of aircraft routing and passenger itinerary networks. For instance, passengers in 
Table 2 Planned passenger itineraries

\begin{tabular}{lcclcc}
\hline Itinerary & $\begin{array}{c}\text { \# of pass. } \\
\text { economy }\end{array}$ & $\begin{array}{l}\text { \# of pass. } \\
\text { business }\end{array}$ & Itinerary & $\begin{array}{c}\text { \# of pass. } \\
\text { economy }\end{array}$ & $\begin{array}{c}\text { \# of pass. } \\
\text { business }\end{array}$ \\
\hline 1 & 183 & 19 & 4 & 113 & 15 \\
$1-2$ & 33 & 4 & 5 & 65 & 13 \\
$1-2-10$ & 12 & 2 & $5-6$ & 90 & 8 \\
$1-6$ & 42 & 5 & 6 & 57 & 6 \\
2 & 153 & 18 & 7 & 130 & 16 \\
$2-10$ & 72 & 6 & $7-8$ & 113 & 11 \\
3 & 67 & 7 & 8 & 51 & 5 \\
$3-4$ & 137 & 13 & 9 & 234 & 26 \\
$3-8$ & 3 & 0 & 10 & 103 & 10 \\
\hline
\end{tabular}

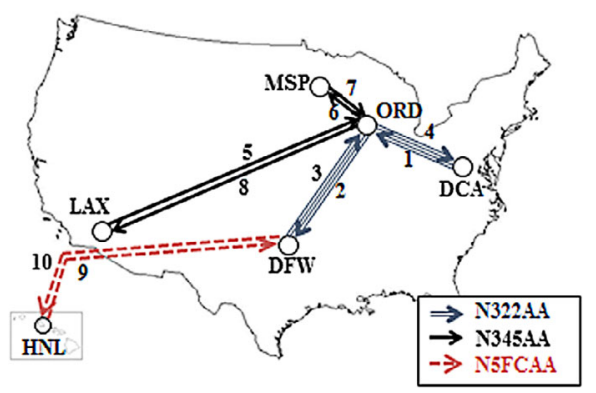

(a) Aircraft Routings

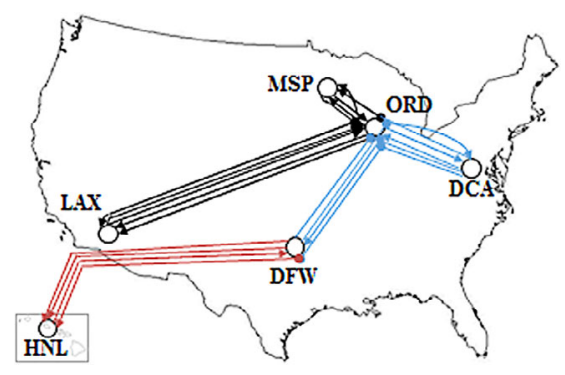

(b) Passenger Itineraries

Fig. 2 Space networks

itinerary 1-2-10 are three-flight passengers who travel from DCA to ORD, ORD to DFW, and finally DFW to HNL. At DFW, passengers in this itinerary switch from N322AA to N5FCAA. Aircraft schedules must provide sufficient time for connected flights, which is called minimum connection time. In this example, minimum connection times are all set to 30 minutes. When the minimum connection time is not satisfied, the itinerary is said to be disrupted. Spill costs of all itineraries in this example are $\$ 50$ and $\$ 200$ for economy and business class passengers, respectively. Delay costs are separated into two terms: delay cost for aircraft and delay cost for passengers. Aircraft delay cost contains several components such as maintenance cost, flight crew and cabin crew cost, and marginal depreciation or lease cost per flight minute. On the other hand, passenger delay cost is the estimated cost of time lost by passengers. Aircraft delay costs are all set to $\$ 20$ per minute; and passenger delay costs per passenger minute are set to $\$ 0.05$ and $\$ 2$ for economy and business class passengers, respectively.

In the given scenario, N322AA is facing maintenance problems before its first flight, and hence, it cannot depart before 09:50. In other words, departure time of flight 1 is delayed by two hours. A common recovery strategy for such disruptions is to postpone all flights until their assigned aircraft are ready. This strategy is known as push-back recovery plan (PB1). A variation of $\mathrm{PB} 1$ is push-back recovery plan maintaining passenger connections (PB2) which may be a good idea when spill costs overweigh delay costs. In PB2, none of the itineraries are disrupted since the departures of all flights are postponed until aircraft 
and passengers are ready. On the other hand, more flights are delayed and greater delay propagation is experienced.

In this study, we propose a new approach with the objective of finding minimum cost recovery plans by deciding on which flights to postpone and how much to postpone, which itineraries to disrupt, cruise times of which flights to compress and how much to compress, and the aircraft of which flights to swap. This integrated approach will be named as aircraftpassenger recovery (APR). Swapping aircraft may provide opportunities to mitigate delays; however, the characteristics of the aircraft should be considered while swapping aircraft. In particular, different types of aircraft may have different fuel efficiencies; and hence, flying the same flight leg with different aircraft may result in different fuel costs. For simplicity, all aircraft in the example have similar fuel efficiencies, and fuel cost of a flight is computed by $0.25 \times \mathrm{Distanc} \mathrm{D}^{2.5} / \mathrm{crt}^{1.5}$, where $\mathrm{crt}$ is the cruise time of the flight. Compression limitations may also vary as displayed in Table 1. Finally, aircraft may have different seat capacities. Therefore, a swap decision may result in spilled passengers due to insufficiency in seat capacity. In the example, capacities of aircraft N322AA, N345AA and N5FCAA are 270-30, 243-27 and 234-26 for economy and business class passengers, respectively. When the aircraft of two flights are swapped, a swap cost is incurred for returning to the original schedule at the end of the day.

Three recovery schemes are displayed in Fig. 3. Lines in grey color represent the flights in the original schedule. In Fig. 3a, it can be seen that only flights in the routing of N322AA are delayed with PB1 recovery policy. Total delay is 420 minutes, resulting in a delay cost of $\$ 36,374$. On the other hand, the connection between flights 2 and 10 , and the connection between 3 and 8 are disrupted. Therefore, the passengers in itineraries 1-2-10, 2-10 and 3-8 are spilled. A total of 87 economy and 8 business class passengers are spilled, and the resulting total spill cost is $\$ 5,950$. As given in Fig. $3 \mathrm{~b}$, in PB2 recovery policy, all connections are maintained; however, in addition to flights in the disrupted routing, flights 8 and 10 are also delayed. Total delay is 570 minutes and the corresponding delay cost is $\$ 45,676$.

Figure $3 c$ presents the solution obtained by APR approach. APR utilizes the swap opportunity between flights 2 and 6 . In the recovery scheme, N322AA operates flights 1, 6, 7 and 8, while N345AA operates flights 5,2, 3 and 4. Resulting swap cost is $\$ 5,000$. In order not to delay flight 2 , cruise time of flight 5 is compressed by 10 minutes so that it arrives 30 minutes (turn time) before the scheduled departure of flight 2. It is important to note that flight 5 is not originally affected from this disruption, but its cruise time is compressed (in albeit of additional fuel cost) due to the swapping decision. Such an interaction will not be foreseen without a global optimization model such as the one proposed in this study. The current industry solution of cost index ratio is useful but the flight crew may not be able to see the consequences of their local decisions. In this solution, passengers in itineraries 1-2 and 1-2-10 are disrupted; and hence, 45 economy and 6 business class passengers are spilled. APR captures the change in aircraft capacities due to the swap; 7 economy and 1 business class passengers are spilled from flight 4 . Resulting spill cost is $\$ 4,000$. Delay is not propagated and only flight 1 is delayed. Moreover, cruise time of flight 1 is compressed by 4.8 minutes leading a total delay of only 115.2 minutes. Corresponding total delay cost is $\$ 10,771$. Speeding up two flights costs $\$ 5,757$ for increased fuel consumption. Some performance measures and cost terms of all recovery schemes are summarized in Table 3 . It can be observed from the cost values that APR finds the optimal tradeoff between the cost terms by optimizing a unified objective function. In this example, a $40 \%$ reduction in total recovery costs with respect to those of $\mathrm{PB} 1$ and $\mathrm{PB} 2$ schemes is provided. 


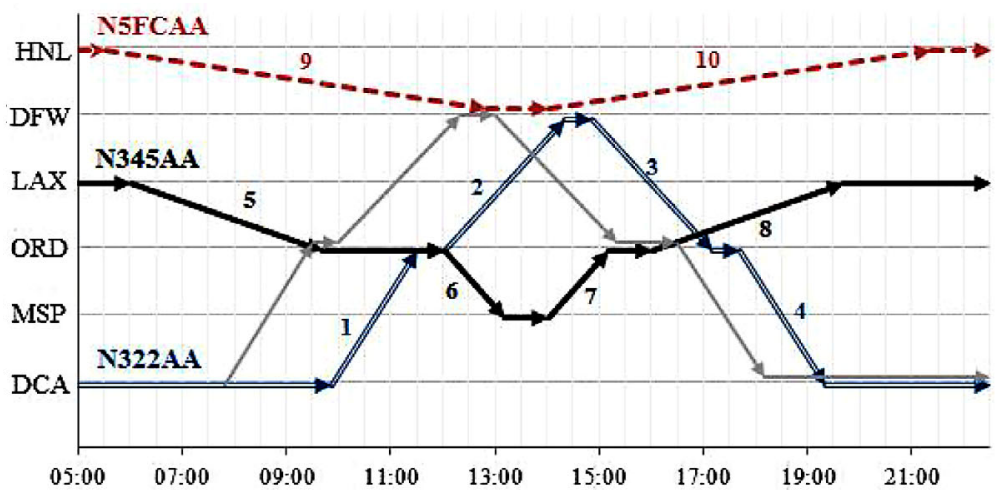

(a) PB1

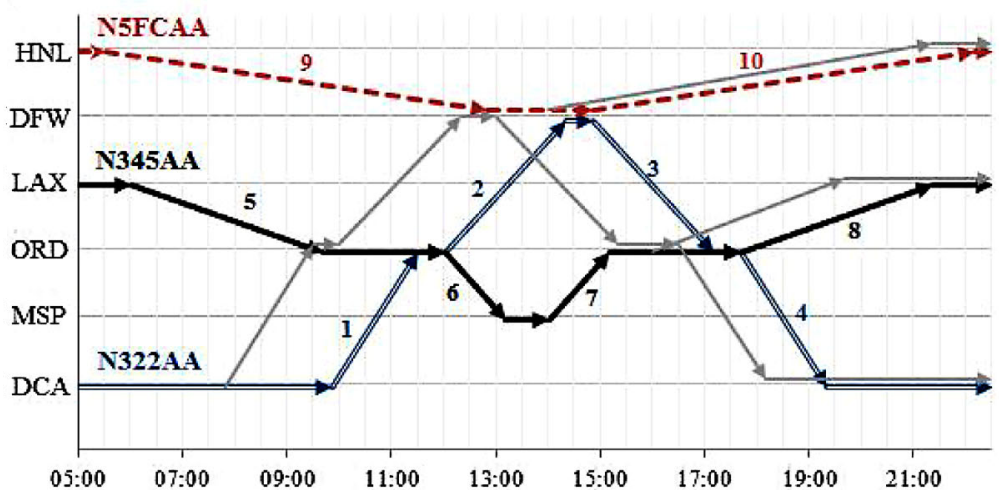

(b) PB2

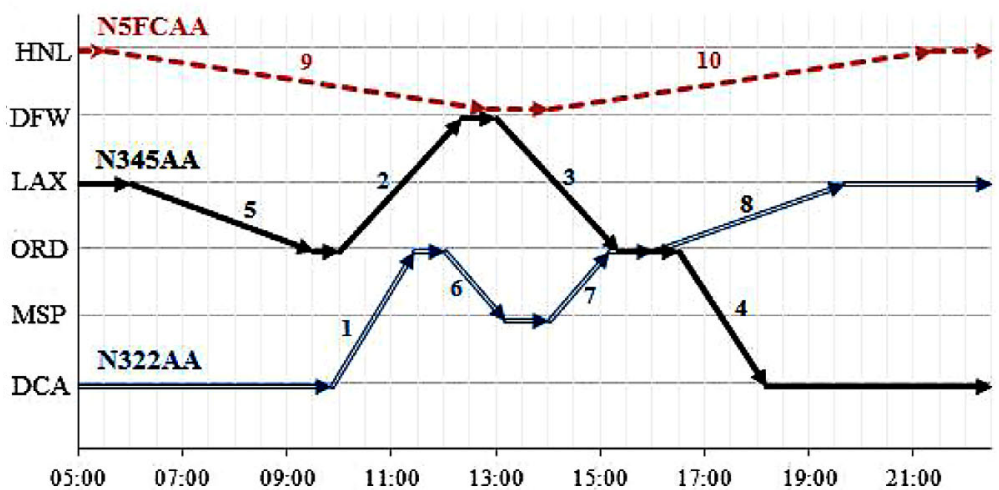

(c) APR

Fig. 3 Recovery schemes

\section{Mathematical formulation}

The objective of aircraft and passenger recovery problem is to find the minimum cost recovery plan given aircraft routings and aircraft characteristics, passenger itineraries and number 
Table 3 Performance measures of solutions

\begin{tabular}{lrrc}
\hline Performance measure & PB1 & PB2 & APR \\
\hline \# of delayed flights & 4 & 6 & 1 \\
Total delay (min.) & 420 & 570 & 115.2 \\
\# of disrupted itineraries & 3 & 0 & 2 \\
\# of spilled passengers & 95 & 0 & 59 \\
Total compression (min.) & 0 & 0 & 14.8 \\
Aircraft delay cost & 8,400 & 11,400 & 2,304 \\
Passenger delay cost & 27,974 & 34,276 & 8,467 \\
Total spill cost & 5,950 & 0 & 4,000 \\
Additional fuel cost & 0 & 0 & 5,757 \\
Swap cost & 0 & 0 & 5,000 \\
Total recovery cost & 42,324 & 45,676 & 25,528 \\
\hline
\end{tabular}

of assigned passengers of each passenger class, and a set of departure or arrival delays of any severity. The decisions include which flights to postpone and how much to postpone, which passenger itineraries to maintain and which to cancel, aircraft of which routings to swap and which flights to speed up. Objective function consists of five cost terms: delay cost for aircraft and passengers, spill cost, swap cost and additional fuel cost. Derivation of the general expression for the nonlinear fuel cost will be given in this section, and conic quadratic reformulation will be explained in Sect. 4 .

\subsection{Fuel cost function}

Since we aim to use cruise speed control of flights in recovery, a realistic fuel cost function is required. In the technical documentations of Airbus (1998) and Boeing (2007), it is reported that direct operating costs of a flight consists of a fixed cost, and variable fuel and time related costs depending on cruise speed and time. However, in aircraft and passenger recovery problem, time related costs of flights should be evaluated considering the superimposition of aircraft and passenger networks instead of considering flights separately. Therefore, we calculate these costs in the mathematical model as aircraft and passenger delay costs. On the other hand, we develop the fuel cost function of a flight with respect to the technical report of Airbus (2004). It is reported that airlines generally operate their aircraft at maximum range cruise (MRC) speeds that result in minimum fuel consumption. The relation of fuel cost with deviation from MRC speed is increasing and convex as displayed in Fig. 4. In (1), we express actual fuel cost of flight $k\left(f c_{k t}\right)$ when operated with the aircraft of routing $t$ as the deviation from the planned (minimum) fuel cost $\left(F C_{k t}\right)$, where $V_{k t}$ is the MRC speed, $\Delta v_{k}$ is the increase in the cruise speed and $K_{k t}$ is flight and aircraft related parameter.

$$
f c_{k t}\left(\Delta v_{k}\right)=F C_{k t} \times\left(\frac{V_{k t}+\Delta v_{k}}{V_{k t}}\right)^{K_{k t}}
$$

where $K_{k t} \geq 1$.

In (2) we express the relation between the cruise speed ( $\left.v_{k}\right)$ and cruise time $\left(\operatorname{crt}_{k}\right)$ of flight $k$, where $D I S T_{k}$ is the cruise stage distance. A similar approximation was also employed in Lovegren and Hansman (2011) to analyze the potential of cruise fuel burn savings.

$$
\operatorname{DIST}_{k}=v_{k} \times \operatorname{crt}_{k}
$$


Fig. 4 Cruise speed-fuel cost relation

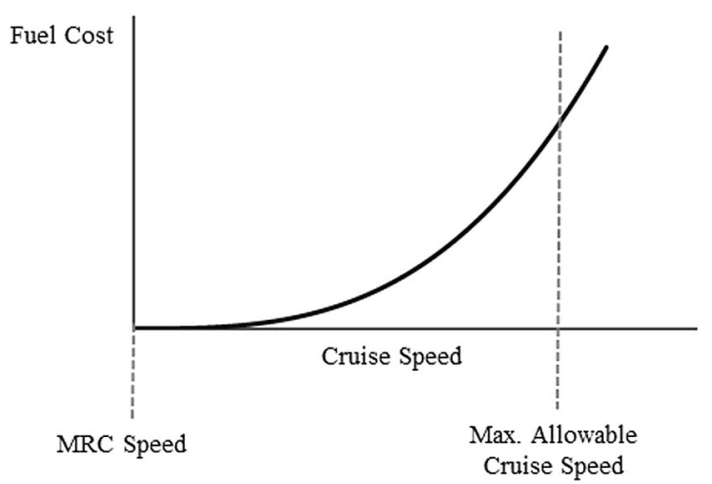

Using relations in (1) and (2), Proposition 1 expresses fuel cost as a function of cruise time.

Proposition 1 Cruise stage fuel cost of flight $k$, when operated with the aircraft of routing $t\left(f c_{k t}\right)$ and when the cruise time is compressed by $\Delta t_{k}$, can be expressed as a function of the amount of compression as follows:

$$
f_{k t}\left(\Delta t_{k}\right)=F C_{k t} \times T_{k t}^{K_{k t}} \times\left(\frac{1}{T_{k t}-\Delta t_{k}}\right)^{K_{k t}}
$$

where $T_{k t}$ is the cruise time of the flight at planned cruise speed.

Proof Using the relation in (2), we have both $D I S T_{k}=V_{k t} \times T_{k t}$ and $D I S T_{k}=\left(V_{k t}+\Delta v_{k}\right) \times$ $\left(T_{k t}-\Delta t_{k}\right)$. Therefore, increase in cruise speed can be expressed in terms of the amount of compression.

$$
\Delta v_{k}=\frac{V_{k t} \times \Delta t_{k}}{T_{k t}-\Delta t_{k}}
$$

Substituting (4) in (1), actual cruise stage fuel cost of a flight can be expressed in terms of the amount of compression in the cruise time.

\subsection{Notation}

Before going through the formulation, notation used throughout this paper is described below.

Sets:

$F \quad$ Set of all scheduled flights

$R \quad$ Set of all scheduled aircraft routings

$F_{t} \quad$ Set of flights in $t^{t h}$ aircraft routing $\quad t \in R$

$I \quad$ Set of passenger itineraries

$I(k) \quad$ Set of passenger itineraries including flight $k \quad k \in F$

$F(i) \quad$ Set of flights in passenger itinerary $i \quad i \in I$

$S \quad$ Set of pairs of flights that can be swapped

$D \quad$ Set of flights that have departure or arrival time disruption

$\mathrm{Pre}_{k} \quad$ Set of flights including flight $k$ and all flights that precede flight $k$ in its routing,

C Set of passenger classes 
In general, aircraft of all flights departing from the same airport can be swapped:

$$
S=\left\{(k, m): k, m \in F, m>k, R_{k} \neq R_{m}, O R I_{k}=O R I_{m}\right\}
$$

$R_{k}$ is the tail number of the $k^{\text {th }}$ flight and the parameter $O R I_{k}$ denotes the origin airport of flight $k$. Flight pairs in set $S$ can be determined by considering various factors. If two aircraft have a long time gap between their arrivals to the airport where swap occurs, then such a swap may be excluded from $S$. If a swap has a very high cost, it causes infeasibilities in crew schedules or in aircraft maintenance restrictions, then it should be excluded from $S$ as well.

\section{Parameters:}

$R_{k}$ : $\quad$ Routing (tail) number of flight $k$

$$
\begin{aligned}
& k \in F \\
& k \in F \\
& k \in F \\
& k \in F, t \in R \\
& k \in F, t \in R \\
& k \in F \\
& i \in I, k \in F(i) \\
& t \in R, c \in C \\
& k \in F \\
& i \in I \\
& i \in I, k \in F(i) \\
& i \in I \\
& k \in F, c \in C \\
& k \in I, c \in C \\
& t \in R \\
& k \in F, t \in R \\
& \\
& k \in F, t \in R \\
& t_{1}, t_{2} \in R \\
& k \in F \\
& k \in F, c \in C \\
& i \in I, c \in C \\
& k \in D \\
& k \in D
\end{aligned}
$$$$
A T_{k}: \quad \text { Scheduled arrival time of flight } k
$$

$F T_{k t}$ : Flight time of flight $k$ if it is flown at planned speed with aircraft of routing $t$

$T_{k t}$ : Cruise time of flight $k$ if it is flown at planned speed with aircraft of routing $t$

$\Gamma_{k}: \quad$ Aircraft turn time for flight $k$

$\Theta_{i k}$ : Minimum connection time for passengers in itinerary $i$ before flight $k$

$C A P_{t}^{c}$ : Seat capacity for passengers of class $c$ in the aircraft assigned to the routing $t$

APre $_{k}$ : Flight that immediately precedes flight $k$ in its routing

First $_{i}$ : First flight in passenger itinerary $i$

$\epsilon_{i}$ : Difference between the ready time of passengers in itinerary $i$ and scheduled

NPass $_{k}^{c}$ : Total number of class c passengers in flight $k$

$N P_{i}^{c}: \quad$ Number of class $c$ passengers in itinerary $i$

$K_{k t}$ : Fuel cost exponent of aircraft of routing $t$ for operating flight $k$

$A l l_{k t}$ : Percentage of cruise time of flight $k$ that can be compressed with aircraft of routing $t$

$F C_{k t}$ : Minimum (planned) fuel cost of flight $k$ if operated with aircraft of routing $t$

$Y_{t_{1} t_{2}}$ : Swap cost incurred if the aircraft of routings $t_{1}$ and are swapped $t_{2}$

$A D C_{k}$ : Aircraft delay cost per flight minute of aircraft

$P D C_{k}^{c}$ : Passenger delay cost per minute per passenger of class $c$ for flight $k$

$S C_{i}^{c}: \quad$ Spill cost per passenger of class $c$ in itinerary $i$

$D D_{k}$ : Amount of departure delay of disrupted flight $k$

$M$ : $\quad$ A sufficiently large amount of time

\section{Decision Variables:}

$d t_{k}: \quad$ Departure time of flight $k$ in the recovered schedule

$$
\begin{aligned}
& k \in F \\
& k \in F \\
& k \in F \\
& k \in F \\
& i \in I, c \in C \\
& k \in F, c \in C \\
& i \in I \\
& (k, m) \in S \\
& k \in F, t \in R
\end{aligned}
$$

\subsection{Constraints}

Constraints of the aircraft and passenger recovery problem are discussed in six groups. 


\subsubsection{Swap constraints}

We limit the number of swaps for the flights in a routing to one in (5) and introduce the auxiliary variable $y_{k t}$ in (6) and (7) in order to indicate that a flight is swapped or not.

$$
\begin{aligned}
\sum_{k \in F_{t}}\left(\sum_{m:(k, m) \in S} x_{k m}+\sum_{m:(m, k) \in S} x_{m k}\right) \leq 1 \quad t \in R \\
\sum_{i \in \operatorname{Pre}_{k}}\left(\sum_{\substack{j:(i, j) \in S, j \in F_{t}}} x_{i j}+\sum_{\substack{j:(i, j) \in S, j \in F_{t}}} x_{j i}\right)=y_{k t} \quad k \in F, t \in R \backslash\left\{R_{k}\right\} \\
1-\sum_{i \in \operatorname{Pre}_{k}}\left(\sum_{j:(i, j) \in S} x_{i j}+\sum_{j:(i, j) \in S} x_{j i}\right)=y_{k R_{k}} \quad k \in F
\end{aligned}
$$

The auxiliary variable $y_{k t}$ takes value one if flight $k$ is operated with the aircraft of routing $t$. Value of $y_{k R_{k}}$ will be equal to one if the flight is not swapped; i.e., if the flight is operated with the originally scheduled aircraft.

\subsubsection{Departure time constraints}

Departure time constraints are developed using the idea that an operation cannot start until all necessary resources are available. Constraints allow early and late departures in order to create a greater feasible region; however, operational restrictions on departure and arrival times can easily be inserted into the model.

$$
\begin{aligned}
& d t_{k} \geq\left(D T_{k}-\epsilon_{i}\right) \times\left(1-z_{i}\right) \quad i \in I, k=\text { First }_{i} \\
& d t_{k} \geq\left(a t_{j}+\Theta_{i j}\right)-M \times z_{i} \quad i \in I, k \in F(i) \backslash \text { First }_{i}, j=\text { PPre }_{i k} \\
& d t_{k} \geq\left(a t_{j}+\Gamma_{j}\right)-M \times\left(\sum_{m:(k, m) \in S} x_{k m}+\sum_{m:(m, k) \in S} x_{m k}\right) \quad k \in F, j=\text { APre }_{k} \\
& d t_{k} \geq\left(a t_{j}+\Gamma_{j}\right)-M \times\left(1-x_{k m}\right) \quad k \in F, j=\text { APre }_{m} \text { where }(k, m) \in S \\
& d t_{k} \geq\left(a t_{j}+\Gamma_{j}\right)-M \times\left(1-x_{m k}\right) \quad k \in F, j=\text { APre }_{m} \text { where }(m, k) \in S
\end{aligned}
$$

We allow flights to depart before the ready time of its passengers. Although it seems an undesirable situation, it might be necessary due to a swapping decision. In such a case, constraint (8) ensures that the passengers having that flight as the first flight in their itineraries are spilled. Passenger connections are modeled with constraint (9). If a flight departs before passengers who have a preceding flight in their itineraries are ready $\left(a t_{j}+\Theta_{i j}\right)$, the variable $z_{i}$ takes value one denoting that the connection is disrupted. Constraint (10) satisfies the aircraft flow balance of two successive flights in a routing considering turn times if the aircraft of these flights are not swapped. In case of swaps, balance is satisfied with constraints (11) and (12). For instance, consider constraint (11) and suppose that the aircraft of flights $k$ and $m$ are swapped. Then, the constraint states that flight $k$ cannot depart before the ready time of the aircraft after flight $j$ which is the predecessor of flight $m$ in its routing in the original schedule. 


\subsubsection{Arrival time and delay constraints}

If the cruise speed is not controlled, arrival time of a flight would be expressed as the sum of the departure time and the planned flight time. Taking compression and swap decisions into consideration, arrival time constraint is given in (13). Since customer convenience is related with on-time arrival performance of flights, we express delay as the difference between actual and planned arrival time in (14). In order not to promote early arrivals, we force delay to be nonnegative in (15).

$$
\begin{aligned}
a t_{k} & \geq d t_{k}+\left(\sum_{t \in R} F T_{k t} \times y_{k t}\right)-\Delta t_{k} \quad k \in F \\
\operatorname{delay}_{k} & \geq a t_{k}-A T_{k} \quad k \in F \\
\text { delay }_{k} & \geq 0 \quad k \in F
\end{aligned}
$$

\subsubsection{Allowable compression constraints}

Maximum compression in the cruise time of a flight is limited and we express this limitation as percentage of the cruise time. Limitation on the compression depends both on the aircraft type and the flight. Constraint (16) defines the upper bound for compression in cruise time. We assume that an airline plans its flight times at maximum range cruise speed which gives minimum fuel cost. Therefore, a longer cruise time would be worse both in terms of time and cost. Constraint (17) enforces compression variable to take nonnegative values.

$$
\begin{aligned}
\Delta t_{k} & \leq \sum_{t \in R} T_{k t} \times A l l_{k t} \times y_{k t} \quad k \in F \\
\Delta t & \geq 0 \quad k \in F
\end{aligned}
$$

\subsubsection{Passenger itinerary disruption and capacity shortage constraints}

Constraints (8) and (9) ensure that binary variable $z_{i}$ takes value one if itinerary $i$ is disrupted. Constraint (18) states that passengers in disrupted itineraries are spilled.

$$
s p_{i}^{c} \geq N P_{i}^{c} \times z_{i} \quad i \in I, c \in C
$$

Since we allow swapping aircraft, seat capacities of flights may change and passengers may also be spilled due to insufficient capacity. In capacity shortage cases, airline should decide on which passengers to spill considering different spill costs of different itineraries. For a given flight $k$, number of class $c$ passengers to be spilled due to capacity shortage is given by $\max \left\{0, N P a s s_{k}^{c}-\sum_{t \in R} C A P_{t}^{c} \times y_{k t}\right\}$. These passengers should be selected from the itineraries that include flight $k$, and some of them may already been spilled due to itinerary disruptions. Constraint (19) ensures that number of passengers assigned to each flight do not exceed the seat capacities of its aircraft.

$$
\sum_{i \in I(k)} s p_{i}^{c} \geq N P a s_{k}^{c}-\sum_{t \in R} C A P_{t}^{c} \times y_{k t} \quad k \in F
$$




\subsubsection{Disruption constraints}

In aircraft and passenger recovery problem, we are dealing with delays of any severity. One can represent a disruption by entering departure delay $\left(D D_{k}\right)$ or arrival delay $\left(A D_{k}\right)$ for a flight. Constraint (20) states that a disrupted flight cannot depart before its ready time. Note that the ready time of these flights after disruption is equal to the sum of scheduled departure time and departure delay. Similarly, constraint (21) expresses arrival time disruptions.

$$
\begin{aligned}
& d t_{k} \geq D T_{k}+D D_{k} \quad k \in D \\
& a t_{k} \geq A T_{k}+A D_{k} \quad k \in D
\end{aligned}
$$

\subsubsection{Objective function}

The objective of the problem is to recover from the disruptions with minimum operating and inconvenience costs. Cost of recovery includes five terms: delay cost for aircraft and passengers, spill cost, swap cost, and increase in the fuel cost. Delay costs include flight and passenger related costs. Passenger related delay costs are based on the number of passengers in the original schedule. For each disrupted passenger, a spill cost is incurred. Both disrupted passenger and passenger delay costs depend on passenger classes. For pairs of flights that are swapped, a swap cost is incurred in order to return to the original schedule before the next day of operations. Cruise stage fuel cost of a flight considering both compression and swap decisions is expressed in (3). Using auxiliary variable $y_{k t}$, additional fuel cost of a particular flight, say $k$, is obtained by $\sum_{t \in R} f_{k t}\left(\Delta t_{k}\right) \times y_{k t}-F C_{k R_{k}}$. The objective function corresponding to the recovery cost is defined in (22) and the complete model is given below:

$$
\begin{gathered}
\min \sum_{k \in F} \text { delay }_{k} \times\left(\text { FDC }_{k}+\sum_{c \in C} \text { NPass }_{k}^{c} \times P D C_{k}^{c}\right)+\sum_{c \in C} \sum_{i \in I} S C_{i}^{c} \times s p_{i}^{c} \\
+\sum_{(k, m) \in S} Y_{R_{k} R_{m}} \times x_{k m}+\left(\sum_{k \in F} \sum_{t \in R} f_{k t}\left(\Delta t_{k}\right) \times y_{k t}-F C_{k R_{k}}\right)
\end{gathered}
$$

subject to

Swap constraints

Departure time constraints

Arrival time and delay constraints

Allowable compression constraints

Passenger itinerary disruption and capacity shortage constraints

Disruption constraints

$z_{i} \in\{0,1\}$

$x_{k m} \in\{0,1\}$

$(k, m) \in S$

Binary decisions included in the proposed model are whether to maintain a passenger connection or not, and whether to swap aircraft of pairs of flights or not, as stated above. Note that APR is a MINLP model having a nonlinear objective function. Fuel cost term of the objective function includes products of convex functions with binary variables. In Sect. 4, we linearize the objective function and then show that the resulting model is SOCPrepresentable. Finally, we explain generation of conic quadratic constraints so that the model can be solved with commercial CQMIP solvers. 


\section{Conic quadratic mixed integer programming model}

In the objective function given in Sect. 3.3.7 for each flight-aircraft pair there exists a nonlinear fuel cost term

$$
f_{k t}\left(\Delta t_{k}\right) \times y_{k t}
$$

which is the product of fuel cost function $\left(f_{k t}\right)$ and flight-aircraft assignment variable $\left(y_{k t}\right)$. This term is nonlinear and nonconvex. In the following, we will first move this function to the constraint set, and then show that the resulting constraint can be reformulated in a way to represent a convex set. We next observe that the resulting convex set can be represented using conic quadratic inequalities.

Substituting the function (3) in (23) we get

$$
\Omega_{k t} \times\left(\frac{1}{T_{k t}-\Delta t_{k}}\right)^{K_{k t}} \times y_{k t}
$$

where $\Omega_{k t}=F C_{k t} \times T_{k t}^{K_{k t}}$.

We next introduce an auxiliary variable crt $_{k}$ that represents the actual cruise time of flight $k$ as below:

$$
\mathrm{crt}_{k}=T_{k t}-\Delta t_{k} \quad k \in F \text { and } t \in R
$$

The next step in representing (23) via conic quadratic inequalities is to replace each term (23) in the objective function with the expression $\Omega_{k t} \times q_{k t}$ where $q_{k t}$ is an auxiliary variable and add the following inequality into the constraint set

$$
\frac{y_{k t}}{\operatorname{crt}_{k}^{K_{k t}}} \leq q_{k t}
$$

This reformulation is obviously equivalent to the original one since it is a minimization problem. In its new form the objective is linear but now we have nonlinear constraints in the constraint set.

Next, we first reformulate (26) so that the reformulation will represent the hypograph of the geometric mean of $2^{l}$ nonnegative variables, which is a convex set. In the following, we drop $k, t$ indices of variables and consider $K=k_{1} / k_{2}$ where $k_{1}, k_{2}$ are integers.

Proposition 2 Inequality (26),

$$
\frac{y}{c r t^{K}} \leq q
$$

can be equivalently written as

$$
y^{2^{l}} \leq q^{k_{2}} \times c r t^{k_{1}}
$$

where $l=\left\lceil\log _{2}\left(k_{1}+k_{2}\right)\right\rceil$.

Proof Inequality

$$
\frac{y}{c r t^{K}} \leq q
$$

can be first written as

$$
y \leq \operatorname{crt}^{k_{1} / k_{2}} \times q
$$


taking $k_{2}^{\text {th }}$ power of both sides we get

$$
y^{k_{2}} \leq c r t^{k_{1}} \times q^{k_{2}}
$$

Exploiting the fact that $y$ is a $0-1$ decision variable in the model, the exponent of $y$ can be increased and set to $2^{l}$ and we get:

$$
y^{2^{l}} \leq c r t^{k_{1}} \times q^{k_{2}} \times 1^{\left(2^{l}-k_{1}-k_{2}\right)}
$$

An inequality of the form $r \leq\left(s_{1} s_{2} \cdots s_{2^{l}}\right)^{1 / 2^{l}}$ with $s_{i} \geq 0$ restrictions defines the hypograph of the geometric mean of variables $s_{1}, \ldots, s_{2}$ which is a convex set. Inequality (28) is of the same form with some restrictions on the variables. $k_{1}$ of them are identical and equal to $\mathrm{crt}_{k}$, $k_{2}$ of them equal to $q$ and $\left(2^{l}-k_{1}-k_{2}\right)$ of them equal to 1 .

Proposition 2 shows that inequality (26) can be reformulated as an inequality which defines a convex set, namely a hypograph of geometric mean of $2^{l}$ variables. This leads to the following result.

Proposition 3 Inequality (26),

$$
\frac{y}{c r t_{k}^{K}} \leq q
$$

with restrictions $y \in\{0,1\}$, crt $_{k} \geq 0, q \geq 0$, can be represented using conic quadratic inequalities.

Proof As given in Ben-Tal and Nemirovski (2001), for a positive integer $l$, an inequality of the form

$$
r^{2^{l}} \leq s_{1} s_{2} \cdots s_{2},
$$

for $r, s_{1}, \ldots, s_{2^{l}} \geq 0$, i.e. a hypograph of geometric mean of $2^{l}$ variables, can be expressed equivalently using $O\left(2^{l}\right)$ variables and $O\left(2^{l}\right)$ hyperbolic inequalities of the form

$$
u^{2} \leq v_{1} v_{2}, \quad u, v_{1}, v_{2} \geq 0
$$

Furthermore, each constraint $u^{2} \leq v_{1} v_{2}$ can be written as a conic quadratic constraint

$$
\left\|\left(2 u, v_{1}-v_{2}\right)\right\| \leq v_{1}+v_{2} .
$$

This reformulation enables us to model the MINLP problem initiated in Sect. 3 as a CQMIP problem. The modified model with linear objective function, linear and conic quadratic constraints can be handled by fast algorithms of commercial CQMIP solvers. We finally illustrate the generation of conic quadratic constraints with an example.

Example 1 Suppose that $K=2.5$; and hence, $k_{1}=5$ and $k_{2}=2$ for a particular flightaircraft pairing. By Proposition 2, inequality

$$
\frac{y}{c r t^{2.5}} \leq q
$$


Fig. 5 Illustration of generation of conic quadratic constraints

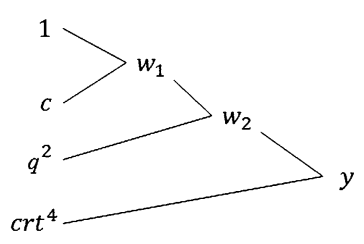

is first expressed as

$$
y^{2} \leq \operatorname{crt}^{5} q^{2}
$$

Then, reformulated as

$$
y^{8} \leq \operatorname{crt}^{5} q^{2} 1^{1}
$$

Obtained inequality can be expressed with the following three inequalities introducing two nonnegative variables:

$$
\begin{aligned}
& w_{1}^{2} \leq c \times 1 \\
& w_{2}^{2} \leq w_{1} \times q \\
& y^{2} \leq w_{2} \times c r t
\end{aligned}
$$

Generation of the inequalities is illustrated in Fig. 5.

These constraints can be represented by the following conic quadratic inequalities:

$$
\begin{aligned}
4 w_{1}^{2}+(c r t-1)^{2} & \leq(c r t+1)^{2} \\
4 w_{2}^{2}+\left(w_{1}-q\right)^{2} & \leq\left(w_{1}+q\right)^{2} \\
4 y^{2}+\left(w_{2}-c r t\right)^{2} & \leq\left(w_{2}+c r t\right)^{2}
\end{aligned}
$$

In the next section, we discuss the computational performance of proposed reformulation.

\section{Computational results}

In order to validate the practicality of the proposed approach for airline and passenger recovery operations, and investigate the effects of several factors on solution quality and performance of the approach with respect to solution time, we have set up an experimental design. Bureau of Transportation Statistics (BTS) provides airline on-time performance data (http://www.transtats.bts.gov/DataIndex.asp). We have extracted the aircraft schedules of a major U.S. airline for three days of operations in April, 2011. Extracted data includes tail numbers, departure and arrival times, flight and cruise times, origins and destinations, and distances of flights. For each aircraft, we randomly selected the number of seats and values of fuel cost parameters. Each flight is included as a single-flight itinerary. Moreover, pair of flights that arrives to a common airport and satisfies minimum passenger connection time are considered as possible passenger connections. The second rule on these pairs to be connecting flights is that the origin of the prior flight should be a different airport than the destination of the latter one. These possible connecting flights are used to generate twoflight passenger itineraries. In a similar manner, again considering the minimum connection times, and origins and destinations of the flights, three-flight itineraries are constructed. 
Number of passengers in each flight is assigned randomly, considering the seat capacities and generated itineraries using these flights. Six different aircraft seat capacities are used: $150,160,180,200,260$ and 300 , where about $10 \%$ of the seats are assigned to business class passengers. Finally, the number of passengers in a flight is allocated randomly to the passenger itineraries that include the flight in consideration. BTS also provides departure delays. Actually disrupted flights are rated with respect to the severity of disturbation on the operations (length of delay, number of succeeding flights in the routing of the disrupted flight, and number of connected flights) and the most severe disruptions are selected for recovery instances.

We have analyzed five factors: number of hubs, number of disruptions, passenger delay cost, fuel cost and swap cost. Number of hubs is directly related to the size of the network in consideration and may affect the performance of the approach. Number of disruptions is another factor affecting the complexity of the problem; and hence, should be analyzed. Instances are created having one, two and five disruptions at a given time frame. Two levels of each of the three cost parameters (passenger delay cost, fuel cost and swap cost parameters) are used in the experimentation. With the increased number of carrier alternatives and increased use of Internet as ticket sale channel, airlines require enhanced methodologies to understand passenger behavior. Modelling passenger behavior is a very important problem in revenue management, and for disruption management as well. In our formulation, passenger behavior in cases of delays is modelled by passenger delay cost parameter. This cost can be regarded as the loss of goodwill cost for the carrier. On the other hand, with the passengers' point of view, it may be regarded as the amount of money a passenger is willing to pay for an arrival with one minute less delay. The value of this parameter may be different for each passenger and it may even vary for an individual in different days, in different times of the day and in different origin-destination pairs. Garrow (2010) presents a novel study that describes the use of choice theory in air transportation for modelling passenger behavior. Garrow et al. (2010) state that from a passenger's perspective, representing the passenger behavior using 'door-to-door' time instead of 'airport-to-airport' time would be more accurate and proposes a formulation to calculate the values of times. On the other hand, Marla et al. (2011) use a passenger delay cost estimated by an airline. In our computational experiments, we have used these estimates proposed by Marla et al. (2011), such that passenger delay cost is set to $\$ 1.09$ and $\$ 1.5$ per minute per passenger for the low and high values, respectively. There are studies in literature that model the behavior of business class passengers, such as Graham et al. (2010). The authors study ticketing, refund and exchange behavior of business class passengers depending on factors such as frequency of travel, carrier, time from ticket purchase and time before flight departure. In terms of delay costs for business class passengers, we can intentionally state that value of time is greater than that for economy class passengers. As the cost estimates proposed by Bratu and Barnhart (2006) suggest, we have doubled the delay costs for business class passengers. We have included a small flight delay cost of $\$ 10$ to prevent unnecessary delays. For low and high values of swap cost, we have used $\$ 500$ (proposed by Marla et al. 2011) and $\$ 1000$, respectively. Low and high fuel cost constants are generated from $\mathrm{U}(0.5,1)$ and $\mathrm{U}(1.5,3)$, respectively, where $\mathrm{U}(a, b)$ is a uniform distribution in interval $(a, b)$. Fuel cost exponents vary from 1.5 to 3.5 . Spill costs are set to $\$ 457.8$ that is evaluated by the method proposed by Marla et al. (2011). For high and low levels of the factors, + and - signs will be used. 15 instances are created for each of the 72 experimental settings. Therefore, a total of 1080 instances are solved using version 12.1 of IBM ILOG CPLEX. In order to test the practicality of our approach, solution times are limited to 300 seconds.

Generated instances include one, two or four hubs. Sizes of the corresponding networks are displayed in Table 4. Number of routings is obtained from the data by counting the 
Table 4 Effect of number of hubs on the size of the network

Table 5 Effect of \# of hubs and \# of disruptions on solution performance of APR

\begin{tabular}{llll}
\hline \# of hubs & $\begin{array}{l}\text { Average \# of } \\
\text { flights }\end{array}$ & $\begin{array}{l}\text { Average \# of } \\
\text { routings }\end{array}$ & $\begin{array}{l}\text { Average \# of } \\
\text { airports }\end{array}$ \\
\hline 1 & 352 & 99 & 41 \\
2 & 619 & 211 & 49 \\
4 & 1429 & 419 & 76 \\
\hline
\end{tabular}

\begin{tabular}{llcll}
\hline $\begin{array}{l}\text { \# of } \\
\text { hubs }\end{array}$ & $\begin{array}{l}\text { \# of } \\
\text { disruptions }\end{array}$ & $\begin{array}{l}\text { Average } \\
\text { CPU time } \\
\text { (sec.) }\end{array}$ & $\begin{array}{l}\text { Average } \\
\text { gap (\%) }\end{array}$ & $\begin{array}{l}\text { Percentage } \\
\text { of optimal } \\
\text { solutions }\end{array}$ \\
\hline 1 & 1 & 4 & - & 100 \\
& 2 & 7 & - & 100 \\
& 5 & 23 & - & 100 \\
2 & 1 & 6 & - & 100 \\
& 2 & 10 & - & 100 \\
& 5 & 55 & 1.7 & 97 \\
& 1 & 44 & - & 100 \\
& 2 & 72 & - & 100 \\
& 5 & 142 & 3.8 & 82 \\
\hline
\end{tabular}

number of routings that visit the selected hubs. All flights in these routings and all airports visited are relevant to our disruption problem; and hence, are included in the model.

Solution times and gaps are summarized in Table 5. Gaps are calculated by dividing the difference between the best integer solution and best lower bound to the best integer solution. When we increase the number of hubs or disruptions, the corresponding solution times and gaps increase as well. We deal with realistic size problem instances, and still solve 1059 of 1080 test instances to optimality using the proposed APR approach. Solution times are greater than one minute only in four-hub instances; however, average solution time is still less than one minute in general. When we analyze the existing schedules, we observed that the number of disruptions was usually less than two in a given time frame. For this particular case, we could solve all of the problem instances optimally in less than one minute of computation time. This is a very important contribution since we can now use optimization techniques instead of relying on ad hoc approaches for such a critical problem.

Table 6 summarizes characteristics of the recovery plans generated by APR with respect to the levels of cost factors ( + and - signs indicate high and low values, respectively). Magnitude of delay cost has the greatest effect on the recovery plan. It can be seen from the table that in the first four settings, number of delayed flights and total delay are less than those for the last four settings permitting disruptions in more passenger itineraries. APR tries to minimize delay propagation as much as possible swapping more flights and using more cruise time compression when delay cost is set to its high level. Therefore, we do not observe a statistically significant effect of the other cost parameters in these settings. When we consider the last four settings separately, we can observe a small effect of fuel cost on the amount of total compression. We do not observe a similar effect for swap cost; however, we can state that swap opportunities help delay mitigation as the number of swaps is proportional to total delay. 
Table 6 Effects of cost factors on recovery actions with APR

\begin{tabular}{llllllll}
\hline $\begin{array}{l}\text { Delay } \\
\text { cost }\end{array}$ & $\begin{array}{l}\text { Fuel } \\
\text { cost }\end{array}$ & $\begin{array}{l}\text { Swap } \\
\text { cost }\end{array}$ & $\begin{array}{l}\text { \# of flights } \\
\text { with delay }\end{array}$ & $\begin{array}{l}\text { Total delay } \\
(\text { min. })\end{array}$ & \# of spills & \# of swaps & $\begin{array}{l}\text { Total compr. } \\
(\mathrm{min})\end{array}$ \\
\hline+ & + & + & 4.07 & 726 & 57.2 & 1.88 & 165 \\
+ & + & - & 4.02 & 731 & 38.0 & 1.98 & 228 \\
+ & - & + & 4.00 & 718 & 41.1 & 1.92 & 179 \\
+ & - & - & 3.89 & 710 & 39.2 & 1.84 & 187 \\
- & + & + & 4.22 & 764 & 43.8 & 1.42 & 148 \\
- & + & - & 4.10 & 747 & 38.4 & 1.46 & 153 \\
- & - & + & 4.24 & 748 & 39.6 & 1.54 & 168 \\
- & - & - & 4.11 & 734 & 30.6 & 1.78 & 171 \\
\hline
\end{tabular}

Table 7 Comparison of APR with other approaches

\begin{tabular}{lllcc}
\hline \# of hubs & \# of disruptions & PB1 $(\%)$ & PB2 $(\%)$ & APR2 (\%) \\
\hline 1 & 1 & 28.1 & 28.1 & 6.3 \\
& 2 & 30.0 & 203.2 & 7.9 \\
& 5 & 18.5 & 135.1 & 5.2 \\
2 & 1 & 29.2 & 29.5 & 6.5 \\
& 2 & 42.4 & 129.2 & 15.7 \\
& 5 & 18.2 & 180.5 & 4.3 \\
4 & 1 & 31.0 & 31.2 & 9.4 \\
& 2 & 42.0 & 130.6 & 6.8 \\
& 5 & 29.8 & 170.9 & 19.4 \\
& & 28.3 & 130.6 & 8.4 \\
\hline
\end{tabular}

Trying to find the optimal balance between five cost terms, APR is expected to outperform methods PB1 and PB2; however, test instances are also solved with these push-back recovery policies in order to observe the improvement in total recovery costs. Moreover, in our experiments, we have observed that complexity of the problem is greatly depending on the number of swap opportunities. Without swap decision, all test instances are solved to optimality in less than a minute (about 15 seconds on the average). Therefore, we have named this alternative solution procedure as APR2 and included in our analysis. On the other hand, PB1 and PB2 plans are created within a second. Table 7 summarizes the percentage cost deviations of alternative approaches with respect to APR solutions. Deviations are calculated by dividing the cost difference to the recovery cost obtained by APR. Greatly propagating delays, PB2 results in most costly recovery plans. PB1 performs better than PB2, but the cost deviation from APR solution is $28.3 \%$. We also observe that APR2 provides great cost improvements compared to push-back recovery policies. On the other hand, $8.4 \%$ deviation indicates the importance of swap opportunities. It is important to note that even in instances where APR stopped with positive gaps, it provided better solutions than APR2. This finding suggests that swap opportunities should be evaluated even they make the problem more complex. Moreover, including the cruise speed control into the integrated recovery process greatly enhances the solution quality as seen in APR2 compared to PB1 or PB2. 
Table 8 Comparison of APR with APR2 within shorter solution time limits

\begin{tabular}{llcc}
\hline \# of hubs & \# of disruptions & 60 seconds & 120 seconds \\
\hline 1 & 1 & 6.3 & 6.3 \\
& 2 & 7.9 & 7.9 \\
& 5 & 5.2 & 5.2 \\
2 & 1 & 6.5 & 6.5 \\
& 2 & 15.7 & 15.7 \\
& 5 & -1.4 & 4.2 \\
4 & 1 & 5.3 & 9.4 \\
& 2 & -4.9 & 6.8 \\
& 5 & -6.2 & 4.8 \\
Average & & 2.1 & 6.9 \\
\hline
\end{tabular}

Since APR2 finds the optimal solution in very short solution times compared to APR, it may be preferred to APR in scenarios with less available solution times. In order to compare solution qualities of these two approaches within shorter solution times, we have experimented same instances with time limits of one and two minutes. Table 8 summarizes the percentage cost deviations of APR2 with respect to APR within 60 and 120 seconds. Considering overall deviations, we can state that APR still outperforms APR2. However, in five disruptions scenario of two-hub case and more than one disruption scenarios of four-hub cases, the deviations are negative when we need to take recovery actions within one minute. The results suggest to use APR2 with these settings. On the other hand, we observe that 120 seconds is sufficient to evaluate and utilize swap opportunities, since APR outperforms APR2 in all settings within two minutes.

\section{Conclusions}

We develop a mathematical model for passenger and aircraft recovery problem. We aim to contribute to the literature by integrating cruise speed control with other recovery actions such as retiming departure and arrival times, and swapping aircraft. Airlines generally operate their flights at cruise speeds that result in minimum fuel consumptions, which is lower than the maximum speed that the aircraft can reach. Our experiments have shown that cruise time compression with cruise speed control can greatly mitigate delays. On the other hand, fuel consumption increases as the aircraft speed up. In accordance with the airline manufacturers' technical specifications, we present a convex and increasing function to express the change in fuel cost as the speed increases. Proposed formulation of the problem is originally a mixed integer nonlinear programming (MINLP) model. We first linearize the nonlinear cost term in the objective function and then show that the resulting problem is second-order cone programming (SOCP) representable. Finally, we create conic quadratic constraints for the nonlinear constraints to be able to solve the problem with commercial MIP solvers such as IBM ILOG CPLEX. We place special emphasis on passenger-related costs. In addition to itineraries, we also discretize passengers with respect to their classes. Proposed model decides passengers in which itineraries will be delayed and how much; and which passenger itineraries will be disrupted, if necessary. Objective of our model is to minimize total recovery cost in case of disruptions. Recovery cost consist of five terms: flight delay cost, 
passenger delay cost, disrupted itinerary cost, swap cost, and increased fuel cost. Proposed model is able to create minimum cost recovery plans by finding the optimal tradeoff between these cost terms. We have performed an extensive computational study for five factors, i.e., number of hubs, number of disruptions, delay cost, fuel cost, and swap cost. Number of hubs determines the size of the network under consideration; and hence, is significant on solution times and gaps. Number of disruptions that will be handled is also one of the most important factors affecting the complexity of the problem. Due to the nature of the problem we expect to handle one or two disruptions at a given time frame. Our computational experiments have shown that proposed approach is able to handle two disruptions on a four-hub network of a major U.S. airline within less than one minute on the average. Moreover, $97 \%$ of the instances including problems dealing with five disruptions at a given time frame are solved to optimality in real time. In this study, we only allow swapping aircraft that satisfy crew and maintenance related constraints, although this assumption can be relaxed to enlarge the solution space.

\section{References}

AhmadBeygi, S., Cohn, A., Guan, Y., \& Belobaba, P. (2008). Analysis of the potential for delay propagation in passenger airline networks. Journal of Air Transport Management, 14(5), 221-236.

Airbus (1998). Airbus flight operations support and line assistance, 'getting to grips with the cost index'. Airbus Customer Services, Issue 2. http://www.iata.org/whatwedo/Documents/fuel/airbus_cost_index_ material.pdf. Visited July 21, 2012.

Airbus (2004). Airbus flight operations support and line assistance, 'getting to grips with fuel economy’. Airbus Customer Services, Issue 3. http://www.iata.org/whatwedo/Documents/fuel/airbus_fuel_ economy_material.pdf. Visited July 21, 2012.

Barnhart, C., \& Cohn, A. (2004). Airline schedule planning: accomplishments and opportunities. Manufacturing \& Service Operations Management, 6(1), 3-22.

Barnhart, C., Fearing, D., \& Vaze, V. (2012). Modeling passenger travel and delays in the national air transportation system. Working paper, Massachusetts Institute of Technology.

Ben-Tal, A., \& Nemirovski, A. (2001). Lectures on modern convex optimization: analysis, algorithms, and engineering applications. Philadelphia: SIAM.

Boeing (2007). Fuel conservation strategies: cost index explained. AERO Quarterly Quarter, 2, 26-28.

Bratu, S., \& Barnhart, C. (2006). Flight operations recovery: new approaches considering passenger recovery. Journal of Scheduling, 9, 279-298.

Clausen, J., Larsen, A., Larsen, J., \& Rezanova, N. J. (2010). Disruption management in the airline industryconcepts, models and methods. Computers \& Operations Research, 37, 809-821.

Cook, A., Tanner, G., Williams, V., \& Meise, G. (2009). Dynamic cost indexing-managing airline delay costs. Journal of Air Transport Management, 15, 26-35.

Garrow, L. A. (2010). Discrete choice modelling and air travel demand: theory and applications. Aldershot: Ashgate Publishing.

Garrow, L. A., Jones, S. P., \& Parker, R. A. (2010). How much airline customers are willing to pay: an analysis of price sensitivity in online distribution channels. Journal of Revenue and Pricing Management, 6(1), $1-20$.

Gopalan, R., \& Talluri, K. T. (1998). Mathematical models in airline schedule planning: a survey. Annals of Operations Research, 76(1), 155-185.

Graham, R. J., Garrow, L. A., \& Leonard, J. D. (2010). Business travelers' ticketing, refund, and exchange behavior. Journal of Air Transportation Management, 16(4), 196-201.

Jafari, N., \& Zegordi, S. H. (2010). The airline perturbation problem: considering disrupted passengers. Transportation Planning and Technology, 33(2), 203-220.

Jarrah, A. I. Z., Yu, G., Krishnamurthy, N., \& Rakshit, A. (1993). A decision support framework for airline flight cancellations and delays. Transportation Science, 27(3), 266-280.

Lan, S., Clarke, J.-P., \& Barnhart, C. (2006). Planning for robust airline operations: optimizing aircraft routings and flight departure times to minimize passenger disruptions. Transportation Science, 40(1), 1528 .

Lovegren, J. A., \& Hansman, R. J. (2011). Quantification of fuel burn reduction in cruise via speed and altitude optimization strategies. Tech. Rep. ICAT-2011-03, Massachusetts Institute of Technology, International Center for Air Transportation (ICAT). 
Kohl, N., Larsen, A., Larsen, J., Ross, A., \& Tiourine, S. (2007). Airline disruption managementperspectives, experiences and outlook. Journal of Air Transport Management, 13(3), 149-162.

Marla, L., Vaaben, B., \& Barnhart, C. (2011). Integrated disruption management and flight planning to trade off delays and fuel burn. Tech. Rep., DTU Management, 2011.

Petersen, J. D., Sölveling, G., Clarke, J.-P., Johnson, E. L., \& Shebalov, S. (2012). An optimization approach to airline integrated recovery. Transportation Science, 46(4), 482-500.

Rosenberger, J. M., Johnson, E. L., \& Nemhauser, G. L. (2003). Rerouting aircraft for airline recovery. Transportation Science, 37(4), 408-421.

Stojkovic, G., Soumis, F., Desrosiers, J., \& Solomon, M. M. (2002). An optimization model for a real-time flight scheduling problem. Transportation Research. Part A, Policy and Practice, 36(9), 779-788.

Thengvall, B. G., Bard, J. F., \& Yu, G. (2000). Balancing user preferences for aircraft schedule recovery during irregular operations. IIE Transactions, 32, 181-193. 